\title{
Retinol effects on CSF outflow through cultured human arachnoid granulation cells: implications for PTC David W Holman ${ }^{1}$, Martin Lubow ${ }^{1}$ and Deborah M Grzybowski*1,2
}

\author{
Address: ${ }^{1}$ Department of Ophthalmology, Neuroophthalmology Research Division, The Ohio State University, College of Medicine, Columbus \\ $\mathrm{OH} 43210, \mathrm{USA}$ and ${ }^{2}$ Department of Biomedical Engineering Center, The Ohio State University, College of Medicine, Columbus OH 43210, USA \\ Email: Deborah M Grzybowski* - grzybowski.3@osu.edu \\ * Corresponding author
}

from 52nd Annual Meeting of the Society for Research into Hydrocephalus and Spina Bifida Providence, RI, USA. II-I 4 June 2008

Published: 3 February 2009

Cerebrospinal Fluid Research 2009, 6(Suppl I):S35 doi:10.I I86/I743-8454-6-SI-S35

This abstract is available from: http://www.cerebrospinalfluidresearch.com/content/6/SI/S35

(c) 2009 Holman et al; licensee BioMed Central Ltd.

\section{Background}

The underlying etiology of pseudotumor cerebri (PTC) remains unknown, however it has been estimated that an identifiable secondary cause is present in up to $10 \%$ of PTC cases. Of these secondary associations, vitamin A is of particular interest, as vitamin A toxicity is associated with an increased intracranial pressure similar to that seen in PTC. Recently, several clinical studies have suggested that serum and CSF retinol levels may be altered in PTC. We hypothesize that increased concentrations of retinoids in the CSF might increase resistance to CSF outflow at the arachnoid granulations (AGs).

\section{Materials and methods}

Cultured human AG cells were exposed to a range of concentrations of all-trans-retinol. Several parameters were measured including viability, proliferation, and protein expression. Viability and proliferation were assessed using commercially available assays. Protein expression was qualitatively examined using immunofluorescent staining for several extracellular matrix and junctional proteins.

\section{Results}

AG cells remained viable over the range of retinol concentrations tested. In addition, retinol decreased AG cell proliferation in a concentration dependent manner. AG cells exposed to $1 \mu \mathrm{M}$ retinol exhibited a statistically significant decrease in proliferation (p-value $<0.01$ ) at 24 and 48 hours compared to controls. AG cells exposed to $1 \mu \mathrm{M}$ retinol for 5 days exhibited no qualitative difference in the expression of the extracellular matrix proteins collagen-IV, laminin, and fibronectin compared to controls.

\section{Conclusion}

We have tested the effects of vitamin A (retinol) on the arachnoidal cells of the CSF outflow pathway. Our results indicate that retinol inhibits growth and proliferation of AGs cells. We have begun to examine if the decrease in proliferation is marked by differentiation and changes in protein expression that might lead to increased resistance to CSF outflow. Future studies will examine the effects of retinol on barrier integrity and outflow resistance of AGs cells. These results have implications for any disorder of CSF homeostasis. 